М.Ю. Федянин ${ }^{1,3 凶}$, e-mail: fedianinmu@mail.ru, Ф.В. Моисеенко ${ }^{2,4,5}$, Д.А. Чекини ${ }^{1}$, В.А. Чубенко ${ }^{2}$, А.С. Жабина ${ }^{2,4}$, Л.А. Загорская ${ }^{2}$, М.М. Крамчанинов ${ }^{2}$, С.А. Тюляндин ${ }^{1}$, В.М. Моисеенко ${ }^{2}$

1 Национальный медицинский исследовательский центр онкологии им. Н.Н. Блохина; 115478, Россия, Москва, Каширское шоссе, д. 24

${ }^{2}$ Санкт-Петербургский клинический научно-практический центр специализированных видов медицинской помощи (онкологический); 197758, Санкт-Петербург, пос. Песочный, Ленинградская ул., д. 68а

3 Российский университет дружбы народов; 117198, Россия, Москва, ул. Миклухо-Маклая, д. 21, корп. 3

${ }^{4}$ Национальный медицинский исследовательский центр онкологии им. Н.Н. Петрова; 197758, Россия, Санкт-Петербург, пос. Песочный, Ленинградская ул., д. 68

${ }^{5}$ Северо-Западный государственный медицинский университет им. И.И. Мечникова; 191015, Россия, Санкт-Петербург, ул. Кирочная, д. 41

\title{
Резюме
}

Введение. Трифлуридин/типирацил (Trifluridine/Tipiracil - FTD/TPI) - новый химиотерапевтический препарат, одобренный более чем в 60 странах для применения у больных метастатическим раком толстой кишки, у которых зарегистрировано прогрессирование или непереносимость лечения фторпиримидинами, оксалиплатином и иринотеканом, анти-VEGR-и анти-EGFRтаргетными агентами. В настоящем исследовании впервые оценена эффективность и переносимость терапии препаратом FTD/TPI в российской популяции пациентов.

Материалы и методы. Проведено подтверждающее открытое однорукавное нерандомизированное исследование в 2 клинических центрах РФ. Основными критериями включения явились: проведение не менее 2-й линии стандартной системной терапии по поводу метастатической аденокарциномы толстой кишки. В качестве первичного критерия эффективности выбрана 2-месячная выживаемость без прогрессирования; вторичными - медиана выживаемости без прогрессирования, частота контроля заболевания, оценка безопасности, общая выживаемость. Номер исследования: NCT03274882.

Результаты. Всего включено в исследование 26 пациентов; медиана возраста составила 60,5 лет (от 30 до 78 ); 19 (73\%) женщин; 4 пациента с ECOG 0 и 22 - с ECOG 1. Всем больным ранее проводилась терапия с включением оксалиплатина, иринотекана, фторпиримидинов, 21 (81\%) - бевацизумаба, 6 (23\%) - анти-EGFR-антитела, 2 (7,7\%) - регорафениба. Медиана курсов лечения составила 4 (1-21), 11 (42,3\%) пациентов находились на лечении 6 и более месяцев. Двухмесячная выживаемость без прогрессирования составила 52\% при медиане выживаемости без прогрессирования 4 мес. (95\% ДИ 1,8-7,4 мес.) Медиана общей выживаемости составила 11 мес. (95\% ДИ 5,2-16,8 мес.). Контроль болезни достигнут у 60\%. Среди нежелательных явлений, связанных с лечением ( $\geqslant 5$ пациентов), превалировали нейтропения, тошнота, рвота, анемия, слабость. Большинство осложнений было 1-2-й ст. Среди нежелательных явлений 3-4-й степени чаще встречалась нейтропения, у 3 пациентов зарегистрирована фебрильная нейтропения 3-й ст.

Выводы. В российской популяции больных химиорефрактерным раком толстой кишки препарат FTD/TPI (TAS-102) показывает эффективность и переносимость, аналогичные регистрационному исследованию RECOURSE.

Ключевые слова: трифлуридин/типирацил, TAS-102, метастатический рак, толстая кишка, химиотерапия, таргетная терапия

Для цитирования: Федянин М.Ю., Моисеенко Ф.В., Чекини Д.А., Чубенко В.А., Жабина А.С., Загорская Л.А., Крамчанинов М.М., Тюляндин С.А., Моисеенко В.М. Подтверждающее исследование эффективности и переносимости терапии трифлуридином/типирацилом (TAS-102) в российской популяции больных химиорефрактерным метастатическим раком толстой кишки. Медицинский совет. 2020;(20):47-52. doi: 10.21518/2079-701X-2020-20-47-52.

Конфликт интересов: авторы заявляют об отсутствии конфликта интересов.

\section{Confirmatory study of the efficacy and tolerability of trifluridine/tipiracil (TAS-102) therapy in the Russian population with chemorefractory metastatic colorectal cancer}

Mikhail Yu. Fedyanin ${ }^{1,3 凶}$, e-mail: fedianinmu@mail.ru, Fedor V. Moiseenko ${ }^{2,4,5}$, Djennet A. Chekini ${ }^{1}$, Viacheslav A. Chubenko², Albina S. Zhabina ${ }^{2,4}$, Lyudmila A. Zagorskaya², Mikhail M. Kramchaninov², Sergei A. Tjulandin ${ }^{1}$, Vladimir M. Moiseyenko²

1 Blokhin National Medical Research Center of Oncology; 24, Kashirskoye Shosse, Moscow, 115478, Russia

2 St Petersburg Clinical Research and Practical Centre of Specialized Types of Medical Care (Oncology); 68a, Leningradskaya St., Pesochnyy Settlement, St Petersburg, 197758, Russia

3 Peoples' Friendship University of Russia; 6, Miklukho-Maklai St., Moscow, 117198, Russia

${ }^{4}$ Petrov National Medical Cancer Research Centre; 68, Leningradskaya St., Pesochnyy Settlement, St Petersburg, 197758, Russia

${ }^{5}$ North-Western State Medical University named after I.I. Mechnikov; 41, Kirochnaya St., St Petersburg, 191015, Russia 


\begin{abstract}
Introduction. Trifluridine/Tipiracil (FTD/TPI) is a new chemotherapeutic drug approved in more than 60 countries for use in patients with metastatic colorectal cancer who have registered progression or intolerance to treatment with fluoropyrimidines, oxaliplatin and irinotecan, anti-VEGR- and anti-EGFR-targeted agents. This study evaluated for the first time the effectiveness and tolerability of FTD/TPI therapy in the Russian patient population.

Materials and methods. A confirmatory open-label single-arm non-randomized trial was conducted in 2 clinical centres in Russia. The main criteria for inclusion were: conduction of at least the 2nd line of standard systemic therapy for metastatic colon adenocarcinoma. The primary efficacy criteria were: 2-month progression-free survival; secondary - median progressionfree survival, disease control frequency, safety assessment, overall survival. Research number: NCT03274882.

Results. A total of 26 patients were included in the study; the median age was 60.5 years (30 to 78); 19 (73\%) women; and 4 patients with ECOG 0 and 22 - with ECOG 1. All patients were previously treated with the inclusion of oxaliplatin, irinotecan, fluoropyrimidines, 21 (81\%) - bevacizumab, 6 (23\%) - anti-EGFR antibodies, and 2 (7.7\%) - regorafenib. The median for treatment courses was 4 (1-21), 11 (42.3\%) patients were treated for 6 months or more. The two-month progression-free survival rate was $52 \%$ with a median progreesion-free survival rate of 4 months ( $95 \% \mathrm{Cl} 1.8-7.4$ months). The median of total survival rate was 11 months (95\% Cl 5,2-16,8 months). Disease control was achieved in 60\%. Neutropenia, nausea, vomiting, anemia, weakness prevailed among undesirable events associated with treatment ( $\geqslant 5$ patients). The majority of complications were of the $1^{\text {st }}-2^{\text {nd }}$ degree. Among the undesirable events of the $3^{\text {rd }}-4^{\text {th }}$ degree, neutropenia was more common, while in 3 patients febrile neutropenia of the 3 rd degree was registered.

Conclusions. In the Russian population of patients with colorectal chemorefractory cancer, the drug FTD/TPI (TAS-102) shows efficacy and tolerability comparable to the RECOURSE registration study.

Keywords: Trifluridine/Tipiracil, TAS-102, metastatic cancer, colorectal, chemotherapy, targeted therapy

For citation: Fedyanin M.Yu., Moiseenko F.V., Chekini D.A., Chubenko V.A., Zhabina A.S., Zagorskaya L.A., Kramchaninov M.M., Tjulandin S.A., Moiseyenko V.M. Confirmatory study of the efficacy and tolerability of trifluridine/tipiracil (TAS-102) therapy in the Russian population with chemorefractory metastatic colorectal cancer. Meditsinskiy sovet = Medical Council. 2020;(20):47-52. (In Russ.) doi: 10.21518/2079-701X-2020-20-47-52.
\end{abstract}

Conflict of interest: the authors declare no conflict of interest.

\section{ВВЕДЕНИЕ}

Трифлуридин/типирацил (Trifluridine/Tipiracil - FTD/ TPI), также известный как TAS-102, представляет собой новый химиотерапевтический таблетированный препарат, одобренный более чем в 60 странах, включая США, Японию, страны Европейского союза, для применения у больных метастатическим раком толстой кишки, у которых зарегистрировано прогрессирование или непереносимость лечения фторпиримидинами, оксалиплатином и иринотеканом, анти-VEGF- и анти-EGFR-таргетными агентами. FTD/TPI включает в себя 2 компонента в отношении 2:1 - трифлуридин - аналог нуклеозида, который после фосфорилирования способен встраиваться в ДНК, вызывая ее дисфункцию, и типирацила гидрохлорид, ингибирующий фермент тимидин фосфорилазу, тем самым увеличивая биодоступность трифлуридина [1]. В проспективном рандомизированном исследовании III фазы в сравнении с плацебо и наилучшей поддерживающей терапией применение нового химиопрепарата улучшило медиану продолжительности жизни с 5,2 до 7,2 мес. (ОР 0,69, р < 0,0001), а одногодичную общую выживаемость - с 16,6 до 27,3\% [2]. И хотя медиана общей выживаемости была выше среди пациентов из Японии, наибольшее снижение риска смерти в сравнении с плацебо зарегистрировано для больных из США, Европы, а затем уже из Японии: ОР 0,56 (95\% ДИ 0,34-0,94), ОР 0,62 (95\% ДИ 0,48-0,80) и ОР 0,75 (95\% ДИ 0,57-1,0) соответственно. Аналогичные тенденции были получены и в отношении выживаемости без прогрессирования. При этом в японской популяции пациентов чаще встречалась анемия 3-4-й ст. (14,1\% против 13\% в США и 23\% в Европе), но реже нейтропения (31,3\% против 29,3\% против 1,7\% соответственно) [3]. Учитывая полученные различия в эффективности и переносимости в различных географических подгруппах пациентов, отсутствие больных из РФ в регистрационном исследовании, была инициирована работа по оценке эффективности и переносимости терапии препаратом FTD/TPI в российской популяции пациентов с химиорефрактерным метастатическим раком толстой кишки.

\section{МАТЕРИАЛЫ И МЕТОДЫ}

Проведено однорукавное нерандомизированное проспективное исследование II фазы в 2 клинических центрах РФ. Регистрационный номер исследования - NCT03274882.

Основными критериями включения пациентов в исследование явились: больные с морфологически подтвержденным метастатическим раком толстой кишки, у которых ранее зарегистрировано прогрессирование или непереносимость терапии с включением фторпиримидинов, оксалиплатина, иринотекана и, при наличии доступа, антиVEGF-препаратов и анти-EGFR-антител при отсутствии мутаций в генах RAS, если такой анализ проводился; ECOG 0-1; возраст старше 18 лет; наличие измеряемых проявлений болезни в соответствии с критериями RECIST 1.1; удовлетворительная функция печени, почек, кроветворения, подписанное информированное согласие.

Режим лечения включал пероральный прием FTD/TPI (TAS-102) в дозе 35 мг/м² х 2 раза в день 1-5, 8-12 дни, 1 раз в 28 дней. Лечение проводилось до прогрессирования или непереносимой токсичности. Оценка эффективности лечения выполнялась 1 раз в 8 нед. до прогрессирования, независимо продолжает прием препарата пациент или 
лечение прекращено не по прогрессированию заболевания. Схема исследования и лечения представлена на рис. 1.

Основным критерием эффективности выбран уровень 2-месячной выживаемости без прогрессирования, оцениваемый как процент больных без прогрессирования и смерти через 2 мес. после начала лечения, в рамках исследования к общему числу пациентов, которые начали терапию. Данный критерий выбран исходя из результатов регистрационного исследования RECOURSE, в котором медиана выживаемости без прогрессирования составила 2 мес. [2].

Вторичными критериями в исследовании явились: выживаемость без прогрессирования, оцениваемая с момента начала лечения до прогрессирования или смерти от любой причины или даты последнего наблюдения; частота контроля болезни, оцениваемой как доля пациентов с объективным эффектом или стабилизацией от общего числа больных; частота объективных эффектов - доля пациентов с объективным эффектом от общего числа больных; время ухудшения функционального статуса пациентов - время, за которое происходило ухудшение показателя ECOG ₹ 2; общая выживаемость, рассчитанная с момента начала лечения до смерти от любой причины или даты последнего наблюдения; частота нежелательных явлений, оцениваемых по критериям NCI-CTCAE 4.03.

Популяция больных для оценки основного критерия эффективности была определена пациентами, которым выполнена как минимум одна оценочная компьютерная томография. При выбывании пациента вследствие смерти от прогрессирования до 1 оценочного визита данное событие расценивалось как прогрессирование. Анализ переносимости терапии исследуемым препаратом проводился на популяции пациентов, у которых был хотя бы один прием FTD/TPI.

Объем выборки пациентов рассчитывался исходя из следующей статистической гипотезы: нулевая гипотеза (Н0): отсутствие прогрессирования заболевания через 2 мес. от начала лечения будет отмечено у 20\% больных; альтерна- тивная гипотеза (H1): отсутствие прогрессирования заболевания через 2 мес. от начала лечения будет зарегистрировано у 50\% больных. При вероятности ошибки І рода 5\%, ошибки II рода - 10\% необходимо включить в исследование 26 пациентов. Результаты исследования будут считаться положительными, если через 2 мес. от начала лечения останется не менее 10 живых пациентов без прогрессирования. Расчет выборки основывался на биноминальном распределении и проводился по формулам из книги Johnson, Kotz and Kemp (1992, equation 3.20) [4] в программе SAS.

При статистическом анализе результатов исследования применялись методы описательной статистики: для непараметрических переменных - число наблюдений, число и процент пациентов с данным признаком; при параметрических - число наблюдений, среднее и стандартное отклонение, медиана, значения 1 и 3 квартилей, минимум и максимум. Выживаемость оценивалась по кривым Каплана - Майера, представлялась в медианах с 95\%-ным доверительным интервалом.

\section{РЕЗУЛЬТАТЫ}

С марта 2017 г. по декабрь 2018 г. в исследование было включено 26 пациентов с медианой возраста 60,5 лет, которые получили хотя бы один прием препарата FTD/TPI. Характеристика пациентов представлена в табл. 1. Большинству пациентов проведено более 4 линий терапии, а длительность метастатической болезни 18 и более месяцев отмечена у 76,9\%. Более 90\% больных имело 3 и более органа, пораженных метастазами, функциональный статус ECOG 0 на момент начала терапии описан только у 15,4\% пациентов.

Медиана числа курсов FTD/TPI составила 4 (1-21), со средней длительностью лечения 25,1 нед., 11 (42,3\%) пациентам лечение проводилось 4 и более месяца. У 13 (52\%) больных потребовалось отсрочить начало хотя бы 1 курса лечения, медиана числа отложенных курсов

Рисунок 1. Схема лечения

Figure 1. Treatment scheme

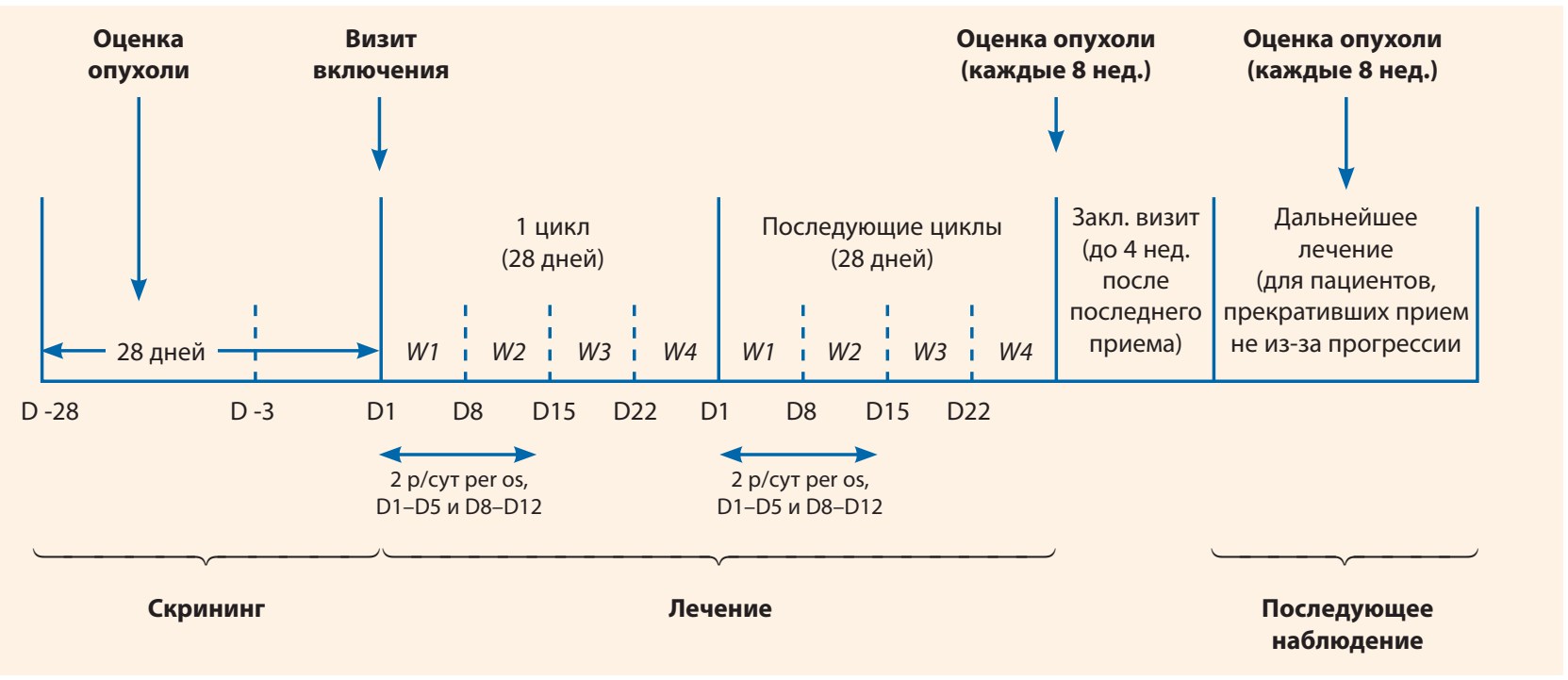


Таблица 1. Характеристика пациентов

Table 1. Characteristics of patients

\begin{tabular}{|c|c|}
\hline Признак & $n(\%)$ \\
\hline Медиана возраста (мин. - макс.) & $60,5(30-78)$ \\
\hline Женский пол & $19(73 \%)$ \\
\hline $\begin{array}{l}\text { ECOG PS: } \\
\cdot 0 \\
-1\end{array}$ & $\begin{array}{c}4(15,4 \%) \\
22(84,6 \%)\end{array}$ \\
\hline $\begin{array}{l}\text { Локализация первичной опухоли: } \\
\text { - Правосторонняя } \\
\text { - Левосторонняя } \\
\text { - Прямая кишки } \\
\text { - Неизвестно }\end{array}$ & $\begin{array}{c}5(19,2 \%) \\
11(42,3 \%) \\
9(34,6 \%) \\
1(3,9 \%)\end{array}$ \\
\hline Мутация в генах RAS & $14(53,8 \%)$ \\
\hline $\begin{array}{l}\text { Длительность метастатической болезни: } \\
\text { • }<18 \text { мес. } \\
\text { • } \geqslant 18 \text { мес. }\end{array}$ & $\begin{array}{c}6(23,1 \%) \\
20(76,9 \%)\end{array}$ \\
\hline $\begin{array}{l}\text { Число органов с метастазами: } \\
\cdot 1-2 \\
\cdot \geqslant 3\end{array}$ & $\begin{array}{c}2(7,7 \%) \\
24(92,3 \%)\end{array}$ \\
\hline $\begin{array}{l}\text { Число предшествующих линий химиотерапии: } \\
\cdot 1 \\
\cdot 2 \\
\cdot 3 \\
\cdot \geqslant 4\end{array}$ & $\begin{array}{c}1(3,9 \%) \\
4(15,4 \%) \\
3(11,5 \%) \\
18(69,2 \%)\end{array}$ \\
\hline $\begin{array}{l}\text { Препараты в предшествующей системной терапии: } \\
\text { - оксалиплатин } \\
\text { - иринотекан } \\
\text { - фторпиримидины } \\
\text { - анти-VEGF } \\
\text { - анти-EGFR } \\
\text { - регорафениб }\end{array}$ & $\begin{array}{l}26(100 \%) \\
26(100 \%) \\
26(100 \%) \\
21(80,8 \%) \\
6(23,1 \%) \\
2(7,7 \%)\end{array}$ \\
\hline
\end{tabular}

ECOG - Eastern Cooperative Oncology Group performance status; VEGF - vascular endothelial growth factor; EGFR - epidermal growth factor receptor.

терапии FTD/TPI составила 1,7, отсрочка начала 4 и более курсов зарегистрирована у 8 (30,8\%) пациентов. Снижение дозы исследуемого препарата на одном из курсов лечения потребовалось 7 (26,9\%) больным.

Среди 25 пациентов, у которых возможно было оценить выживаемость (1 пациент выбыл до выполнения 1 оценочного визита не по прогрессированию), показатель 2-месячной выживаемости без прогрессирования составил 52\%, при медиане выживаемости без прогрессирования 4 мес. (95\% ДИ 1,8-7,4) (рис. 2). Расчетная вероятность выживаемости без прогрессирования на 2, 4 и 6 мес. составила 0,6, 0,5 и 0,4 соответственно. Контроль болезни удалось достичь у 60\% пациентов (95\% ДИ 38,7-78,9). У всех этих 15 пациентов зарегистрирована стабилизация при длительности контроля болезни 8,4 мес. (у 93,3\% - 3 и более месяца).

Медиана общей выживаемости составила 11 мес. (95\% ДИ 5,2-16,8) при показателях 6-, 9- и 12-месячной выживаемости 0,62; 0,58 и 0,46 соответственно (рис. 3).

Медиана времени до ухудшения функционального статуса до уровня ECOG $\geqslant 2$ составила 7,7 мес. Среди 12 пациентов $(46,2 \%)$ с ухудшением статуса ECOG у 2 больных произошло снижение ECOG с 0 до 2, у 6 - с 1 до 2 и у 4 - с 1 до 3. У 13 больных (50\%) функциональный статус не менялся.

Осложнения, связанные с исследуемым препаратом, зарегистрированы у 76,9\% из 26 пациентов. Спектр,
Рисунок 2. График выживаемости без прогрессирования

Figure 2. Progression-free survival rate

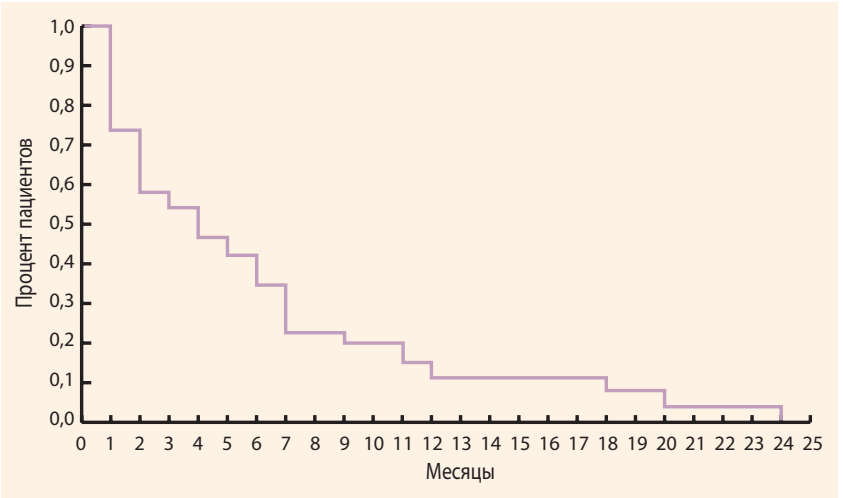

Рисунок 3. График общей выживаемости

Figure 3. Overall survival rate graph

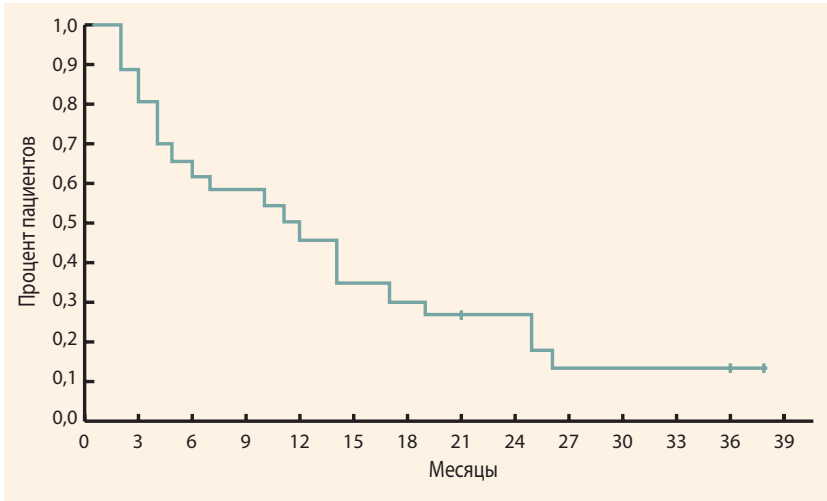

частота и тяжесть нежелательных явлений представлены в табл. 2 (отмечены осложнения с частотой проявления $\geqslant 10 \%$. Наиболее часто осложнения 3-4-й ст. проявлялись нейтропенией (у 3 пациентов зарегистрирована фебрильная нейтропения 3-й ст.), диареей, тошнотой, анемией и слабостью.

Среди 13 (52\%) пациентов, которым потребовалась отсрочка начала очередного курса лечения, у 12 больных в качестве причины фигурировала нейтропения, у 1 анемия. Из 7 (26,9\%) пациентов, которым потребовалось снижение дозы препарата, у 5 снижение дозы сопровождалось отсрочкой начала курса терапии. Только у 2 пациентов (7,7\%) лечение было полностью прекращено в связи с нежелательными явлениями: у 1 больного общее ухудшение состояния 3-й ст., у 2 - прогрессирование основного заболевания. Не отмечено смертей в процессе лечения; 3 пациента умерло в процессе 30 дней наблюдения в связи с прогрессированием.

\section{ОБСУЖДЕНИЕ}

В нашем исследовании более 10 пациентов находились на лечении без прогрессирования более 2 мес., что подтверждает эффективность препарата FTD/TPI и российской популяции больных метастатическим химиореф- 
рактерным раком толстой кишки. Если же сравнить эффективность с результатами регистрационного исследования RECOURSE, то можно отметить лучшие показатели в нашей работе: медиана выживаемости без прогрессирование была в 2 раза выше - 4 мес. против 2 мес. терапии; частота достижения контроля болезни - 60\% против 44\%; медиана общей выживаемости - 11 мес. против 7,2 мес.; медиана времени до ухудшения функционального статуса до уровня ECOG $\geqslant 2$ - 7,2 мес. против 5,7 мес. [5]. Такие различия можно объяснить небольшой выборкой пациентов, участием в исследовании только 2 академических клинических центров, а также превалированием пациентов с длительно текущим метастатическим заболеванием (18 и более месяцев). Последний факт, как известно, в популяции больных с химиорефрактерным раком толстой кишки был ассоциирован с большим числом пациентов без прогрессирования в течение 4-6 мес. как в отношении применения FTD/TPI [6], так и регорафениба [7, 8]. Также данный фактор может отражать медленную скорость роста опухоли, что является некоторым сигналом о возможности с большей вероятностью достичь контроля болезни на препарате FTD/TPI в сравнении с регорафенибом [9].

Рассматривая особенности переносимости FTD/TPI в российской популяции пациентов, отметим, что общее число осложнений и частота нежелательных явлений 3-4 ст. были аналогичными регистрационному исследованию: 76,9\% против 98\% и 65,3\% против 69\% соответственно.

Таблица 2. Нежелательные явления, связанные с исследуемым препаратом

Table 2. Adverse events related to the studied drug

\begin{tabular}{l|c|c}
\multicolumn{1}{c|}{ Нежелательные явления } & $\begin{array}{c}\text { Все степени } \\
\text { n (\%) }\end{array}$ & $\begin{array}{c}\text { 3-4-й степени } \\
\text { n (\%) }\end{array}$ \\
\hline Bсе & $20(76,9 \%)$ & $17(65,3 \%)$ \\
\hline Все серьезные нежелательная явления & $17(65,4 \%)$ & $5(19,2 \%)$ \\
\hline Нейтропения & $18(69,2 \%)$ & $12(46,2 \%)$ \\
\hline Диарея & $8(30,8 \%)$ & 0 \\
\hline Анемия & $8(30,8 \%)$ & $3(11,5 \%)$ \\
\hline Астения & $8(30,8 \%)$ & $1(3,8 \%)$ \\
\hline Снижение аппетита & $8(30,8 \%)$ & 0 \\
\hline Слабость & $7(26,9 \%)$ & $1(3,8 \%)$ \\
\hline Тошнота & $7(26,9 \%)$ & 0 \\
\hline Фебрильная нейтропения & $3(11,5 \%)$ & $3(11,5 \%)$ \\
\hline Рвота & $3(11,5 \%)$ & 0
\end{tabular}

Однако число серьезных нежелательных явлений (SAE) зарегистрировано больше в нашей работе: 65,4\% против 30\%. Последнее можно объяснить тем, что часть SAE были связаны с прогрессированием заболевания, часть в соответствии с протоколом трактовались как не жизнеугрожающие, но значимые с медицинской точки зрения. В спектре осложнений 3-4-й ст., так же как и в популяции США и Европы, лидировала нейтропения при аналогичной частоте фебрильной нейтропении, а частота анемий была приближена к японской популяции пациентов [3].

Еще одним фактором, по которому можно судить о переносимости терапии любым препаратом, это доля пациентов, которым потребовалась редукция дозы или отсрочка начала курсов лечения. Начало курса терапии было отложено в регистрационном исследовании у 53\%, в исследовании в РФ - 52\%; редукция дозы FTD/TPI - у $14 \%$ и 26,9\% соответственно [3]. Такое, практически в 2 раза, увеличение числа случаев редукции препарата, возможно, явилось одним из факторов, ассоциированным с более высокими показатели выживаемости пациентов в РФ [10].

Небольшое число пациентов и нерандомизированный характер исследования ограничили нам возможность проведения поиска прогностических и предикторных маркеров. Тем не менее минимально необходимое число пациентов, проспективный набор больных позволили подтвердить эффективность и переносимость препарата FTD/TPI (TAS-102) в российской популяции пациентов с химиорефрактерным метастатическим раком толстой кишки, аналогичные регистрационному исследованию RECOURSE.

\section{ЗАКЛЮЧЕНИЕ}

Результаты проспективного исследования по применению трифлуридина/типирацила у пациентов с химиорефрактерным метастатическим раком толстой кишки в РФ показали сопоставимую с регистрационными исследованиями эффективность и переносимость препарата. Это позволяет назначать его при прогрессировании после схем с включением окслиплатина, иринотекана, фторпиримидинов, таргетной терапии. И после получения регистрации в РФ препарат может быть рекомендован для включения в клинические рекомендации по лечению метастатического рака толстой кишки как в монорежиме, так, возможно, и в комбинации с бевацизумабом в 3 и последующих линиях лечения $[11,12]$.

Поступила / Received 06.10.2020 Поступила после рецензирования / Revised 23.10.2020 Принята в печать / Accepted 29.10.2020
1. Kish T., Uppal P. Trifluridine/Tipiracil (Lonsurf) for the treatment of metastatic colorectal Cancer. PT. 2016;41(5):314-325. Available at: https:// www.ncbi.nlm.nih.gov/pmc/articles/PMC4849340/

2. Mayer RJ., Ohtsu A., Yoshino T., Falcone A., Garcia-Carbonero R., Tabernero J. et al. TAS-102 versus placebo plus best supportive care in patients with metastatic colorectal cancer refractory to standard therapies: final survival results of the phase III recourse trial. J Clin Oncol. 2016;34(4S):634. doi: 10.1200/jco.2016.34.4_suppl.634.

3. Van Cutsem E., Mayer R.J., Laurent S., Winkler R., Grávalos C., Benavides M. et al. The subgroups of the phase III RECOURSE trial of trifluridine/tip- iracil (TAS-102) versus placebo with best supportive care in patients with metastatic colorectal cancer. Eur J Cancer. 2018;90:63-72. doi: 10.1016/j.ejca.2017.10.009.

4. Johnson N.L., Kotz S., Kemp A.W. Univariate discrete distributions. $2^{\text {th }}$ ed. John Wiley \& Sons; 1992.565 p. Available at: https://ideas.repec.org/a/ eee/csdana/v17y1994i2p240-241.html.

5. Mayer R.,., Van Cutsem E., Falcone A., Yoshino T., Garcia-Carbonero R., Mizunuma N. et al. Randomized trial of TAS-102 for refractory metastatic colorectal cancer. N Engl J Med. 2015;372(20):1909-1919. doi: 10.1056/ NEJMoa1414325. 
6. Cremolini C., Rossini D., Martinelli E., Pietrantonio F., Lonardi S., Noventa S. et al. Trifluridine/Tipiracil (TAS-102) in refractory metastatic colorectal cancer: a multicenter register in the frame of the Italian compassionate use program. Oncologist. 2018;23(10):1178-1187. doi: 10.1634/theoncologist.2017-0573.

7. Garcia-Carbonero R., Van Cutsem E., Ciardiello F., Sobrero A., Banzi C., Barone C. et al. Subgroup analysis of patients with metastatic colorectal cancer (mCRC) treated with regorafenib (REG) in the phase 3b CONSIGN trial who had progression-free survival (PFS) $>4$ months (m). Ann Oncol. 2016;27(4 Suppl.):39-54. doi: 10.1093/annonc/mdw335.27.

8. Grothey A., Falcone A., Humblet Y., Bouche O., Mineur L., Adenis A. et al. Characteristics of patients (pts) with metastatic colorectal cancer (mCRC) treated with regorafenib (REG) who had progression-free survival (PFS) >4 months (m): Subgroup analysis of the phase 3 CORRECT trial. Ann Oncol. 2016;27(6 Suppl.):149-206. doi: 10.1093/annonc/mdw370.64.

9. Masuishi T., Taniguchi H., Kawakami T., Kawamoto Y., Kadowaki S. Onozawa Y. et al. Impact of tumour growth rate during preceding treatment on tumour response to regorafenib or trifluridine/tipiracil in refrac tory metastatic colorectal cancer. ESMO Open. 2019:4(6):e000584. 2016;27(6):506. doi: 10.1136/esmoopen-2019-000584

10. Fernandez Montes F.A., Vazquez Rivera F., Martinez Lago M., Covela Rúa M., Cousillas Castiñeiras A., Gonzalez Villarroel P. et al. Efficacy and safety of trifluridine/tipiracil in third-line and beyond for the treatment of patients with metastatic colorectal cancer in routine clinical practice: patterns of use and prognostic nomogram. Clin Transl Oncol. 2020;22(3):351-359. 2016:27(6):506. doi: 10.1007/s12094-019-02130-x.

11. Kotani D., Kuboki Y., Horasawa S., Kaneko A., Nakamura Y., Kawazoe A. et al. Retrospective cohort study of trifluridine/tipiracil (TAS-102) plus bevacizumab versus trifluridine/tipiracil monotherapy for metastatic colorectal cancer. BMC Cancer. 2019;19(1):1253. doi: 10.1186/s12885-019-6475-6.

12. Pfeiffer P., Yilmaz M., Möller S., Zitnjak D., Krogh M., Petersen L.N. et al. TAS-102 with or without bevacizumab in patients with chemorefractory metastatic colorectal cancer: an investigator-initiated, open-label, randomised, phase 2 trial. Lancet Oncol. 2020;21(3):412-420. 2016;27(6):506 doi: 10.1016/S1470-2045(19)30827-7.

\section{Информация об авторах:}

Федянин Михаил Юрьевич, д.м.н., старший научный сотрудник онкологического отделения лекарственного лечения (химиотерапевтического) №2, Национальный медицинский исследовательский центр онкологии им. Н.Н. Блохина; 115478, Россия, Москва, Каширское шоссе, д. 24; доцент кафедры онкологи и гематологии, Российский университет дружбы народов; 117198, Россия, Москва, ул. Миклухо-Маклая, д. 21, корп. 3; ORCID: 0000-0001-5615-7806; SPIN-код: 4381-5628; e-mail: fedianinmu@mail.ru

Моисеенко Федор Владимирович, д.м.н., доцент, заведующий отделением химиотерапии, Санкт-Петербургский клинический научно-практический центр специализированных видов медицинской помощи (онкологический); 197758, Россия, Санкт-Петербург, пос. Песочный, Ленинградская ул., д. 68а; научный сотрудник научного отдела инновационных методов терапевтической онкологии и реабилитации, Национальный медицинский исследовательский центр онкологии им. Н.Н. Петрова; 197758, Россия, Санкт-Петербург, пос. Песочный, Ленинрадская ул., д. 68; профессор кафедры онкологии, Северо-Западный государственный медицинский университет имени И.И. Мечникова; 191015, Россия, Санкт-Петербург, ул. Кирочная, д. 41; ORCID: 0000-0003-2544-9042; SPIN-код: 7462-2225; e-mail: moiseenkofv@gmail.com Чекини Дженнет Ашеровна, к.м.н., врач-онколог отделения лекарственного лечения (химиотерапевтического) №2, Национальный медицинский исследовательский центр онкологии им. Н.Н. Блохина; 115478, Россия, Москва, Каширское шоссе, д. 24

Чубенко Вячеслав Андреевич, к.м.н., заведующий онкологическим химиотерапевтическим отделением (противоопухолевой лекарственной терапии) солидных опухолей, Санкт-Петербургский клинический научно-практический центр специализированных видов медицинской помощи (онкологический); 197758, Россия, Санкт-Петербург, пос. Песочный, Ленинградская ул., д.68a; ORCID: 0000-0001-6644-6687; e-mail: vchubenko@me.com

Жабина Альбина Сергеевна, к.м.н., врач-онколог онкологического химиотерапевтического (противоопухолевой лекарственной терапии) биотерапии отделения, Санкт-Петербургский клинический научно-практический центр специализированных видов медицинской помощи (онкологический); 197758, Россия, Санкт-Петербург, пос. Песочный, Ленинградская ул., д. 68а

Загорская Людмила Анатольевна, врач-химиотерапевт онкологического химиотерапевтического отделения (противоопухолевой лекарственной терапии) солидных опухолей, Санкт-Петербургский клинический научно-практический центр специализированных видов медицинской помощи (онкологический); 197758, Россия, Санкт-Петербург, пос. Песочный, Ленинградская ул., д. 68а

Крамчанинов Михаил Михайлович, врач-онколог онкологического химиотерапевтического (противоопухолевой лекарственной терапии) биотерапии отделения, Санкт-Петербургский клинический научно-практический центр специализированных видов медицинской помощи (онкологический); 197758, Россия, Санкт-Петербург, пос. Песочный, Ленинградская ул., д. 68а

Тюляндин Сергей Алексеевич, д.м.н., профессор, заведующий онкологическим отделением лекарственного лечения (химиотерапевтического) №2, Национальный медицинский исследовательский центр онкологии им. Н.Н. Блохина; 115478, Россия, Москва, Каширское шоссе, д. 24; ORCID: 0000-0001-9807-2229; e-mail: stjulandin@gmail.com

Моисеенко Владимир Михайлович, д.м.н., профессор, директор, Санкт-Петербургский клинический научно-практический центр специализированных видов медицинской помощи (онкологический); 197758, Россия, Санкт-Петербург, пос. Песочный, Ленинградская ул., д. 68а; ORCID: 0000-0003-4807-7915; e-mail: moiseyenkov@gmail.com

\section{Information about the authors:}

Mikhail Yu. Fedyanin, Dr. of Sci. (Med.), Senior Researcher of the Oncological Department of Medication-assisted Treatment (Chemotherapeutic) No. 2, Blokhin National Medical Research Center of Oncology; 24, Kashirskoye Shosse, Moscow, 115478, Russia; Associate Professor of the Department of Oncology and Hematology, Peoples' Friendship University of Russia; 6, Miklukho-Maklai St., Moscow, 117198, Russia; ORCID: 0000-0001-5615-7806; e-mail: fedianinmu@mail.ru

Fedor V. Moiseenko, Dr. of Sci. (Med.), Associate Professor, head of the Chemotherapy Department, St Petersburg Clinical Research and Practical Centre of Specialized Types of Medical Care (Oncology); 68a, Leningradskaya St., Pesochnyy Settlement, St Petersburg, 197758, Russia; researcher of the Scientific Department of Innovative Methods of Therapeutic Oncology and Rehabilitation, Petrov National Medical Cancer Research Centre; 68, Leningradskaya St., Pesochnyy Settlement, St Petersburg, 197758, Russia; Professor of the Oncology Department, North-Western State Medical University named after I.I. Mechnikov; 41, Kirochnaya St., St Petersburg, 191015, Russia; ORCID: 0000-0003-2544-9042; e-mail: moiseenkofv@gmail.com

Djennet A. Chekini, Cand. of Sci. (Med.), Oncologist of the Oncological Department of Medication-assisted Treatment (Chemotherapeutic) No. 2, Blokhin National Medical Research Center of Oncology; 24, Kashirskoye Shosse, Moscow, 115478, Russia

Viacheslav A. Chubenko, Cand. of Sci. (Med.), Head of the Oncological Chemotherapeutic department (anticancer drug therapy) for solid tumours, St Petersburg Clinical Research and Practical Centre of Specialized Types of Medical Care (Oncology); 68a, Leningradskaya St. Pesochnyy Settlement, St Petersburg, 197758, Russia; ORCID: 0000-0001-6644-6687; e-mail: vchubenko@me.com

Albina S. Zhabina, Cand. of Sci. (Med.), Oncologist of the Oncological Chemotherapeutic (anticancer drug therapy) Biotherapy Department, St Petersburg Clinical Research and Practical Centre of Specialized Types of Medical Care (Oncology); 68a, Leningradskaya St., Pesochnyy Settlement, St Petersburg, 197758, Russia

Lyudmila A. Zagorskaya, Chemotherapist of the Oncological Chemotherapeutic department (anticancer drug therapy) for solid tumours, St Petersburg Clinical Research and Practical Centre of Specialized Types of Medical Care (Oncology); 68a, Leningradskaya St., Pesochnyy Settlement, St Petersburg, 197758, Russia

Mikhail M. Kramchaninov, Oncologist of the Oncological Chemotherapeutic (anticancer drug therapy) Biotherapy Department, St Petersburg Clinical Research and Practical Centre of Specialized Types of Medical Care (Oncology); 68a, Leningradskaya St., Pesochnyy Settlement, St Petersburg, 197758, Russia

Sergei A. Tjulandin, Dr. of Sci. (Med.), Professor, Head of the Oncological Department of Medication-assisted Treatment (Chemotherapeutic) No. 2, Blokhin National Medical Research Center of Oncology; 24, Kashirskoye Shosse, Moscow, 115478, Russia; ORCID: 0000-0001-9807-2229; e-mail: stjulandin@gmail.com

Vladimir M. Moiseyenko, Dr. of Sci. (Med.), Professor, director, St Petersburg Clinical Research and Practical Centre of Specialized Types of Medical Care (Oncology); 68a, Leningradskaya St., Pesochnyy Settlement, St Petersburg, 197758, Russia; ORCID: 0000-0003-4807-7915; e-mail: moiseyenkov@gmail.com 